\title{
Anhang lb: URLs deutschsprachiger (professioneller) Nachrichtenmedien
}

\begin{tabular}{|c|c|c|c|c|c|c|c|}
\hline Land & URL & URL & Land & URL & Land & URL & URL \\
\hline D & 24vest.de & $\begin{array}{l}\text { morgenpost- } \\
\text { abo.de }\end{array}$ & $A$ & achtzig.com & $\mathrm{CH}$ & 1815.ch & $\begin{array}{l}\text { nidwaldner } \\
\text { zeitung.ch }\end{array}$ \\
\hline D & $\begin{array}{l}\text { aachener- } \\
\text { nachrichten.de }\end{array}$ & morgenpost.de & $A$ & advantage.at & $\mathrm{CH}$ & 20min.ch & $\begin{array}{l}\text { noz-oberaargau. } \\
\text { ch }\end{array}$ \\
\hline D & $\begin{array}{l}\text { aachener- } \\
\text { zeitung.de }\end{array}$ & morgenweb.de & $A$ & alpenpost.at & $\mathrm{CH}$ & 20minuten.ch & noz.ch \\
\hline D & abendblatt.de & moz.de & $A$ & apropos.or.at & $\mathrm{CH}$ & 20minutes.ch & nzz.ch \\
\hline D & $\begin{array}{l}\text { abendzeitung- } \\
\text { muenchen.de }\end{array}$ & $\begin{array}{l}\text { msn.com/ } \\
\text { de-de/ } \\
\text { nachrichten }\end{array}$ & $A$ & atv.at/aktuell & $\mathrm{CH}$ & 20minuti.ch & nzzas.nzz.ch \\
\hline D & $\begin{array}{l}\text { aichacher- } \\
\text { zeitung.de }\end{array}$ & mt-news.de & $A$ & augustin.or.at & $\mathrm{CH}$ & 24heures.ch & nzzas.nzz.ch \\
\hline D & $\begin{array}{l}\text { alfelder- } \\
\text { zeitung.de }\end{array}$ & mt.de & $A$ & $\begin{array}{l}\text { badener } \\
\text { zeitung.at }\end{array}$ & $\mathrm{CH}$ & $\begin{array}{l}\text { aarauer-nach- } \\
\text { richten.ch }\end{array}$ & oberaargauer.ch \\
\hline D & all-in.de & $\begin{array}{l}\text { muehlacker- } \\
\text { tagblatt.de }\end{array}$ & $A$ & $\begin{array}{l}\text { bauernzei- } \\
\text { tung.at }\end{array}$ & $\mathrm{CH}$ & $\begin{array}{l}\text { aargauerzeitung. } \\
\text { ch }\end{array}$ & $\begin{array}{l}\text { oberbaselbieter } \\
\text { zeitung.ch }\end{array}$ \\
\hline D & aller-zeitung.de & $\begin{array}{l}\text { muensterland- } \\
\text { zeitung.de }\end{array}$ & $A$ & $\begin{array}{l}\text { boersen- } \\
\text { kurier.at }\end{array}$ & $\mathrm{CH}$ & $\begin{array}{l}\text { affolternanzei- } \\
\text { ger.ch }\end{array}$ & $\begin{array}{l}\text { oberland-nach } \\
\text { richten.ch }\end{array}$ \\
\hline D & $\begin{array}{l}\text { allgemeine- } \\
\text { zeitung.de }\end{array}$ & $\begin{array}{l}\text { muenstersche- } \\
\text { zeitung.de }\end{array}$ & $A$ & bvz.at & $\mathrm{CH}$ & agefi.ch & $\begin{array}{l}\text { obersee-nach- } \\
\text { richten.ch }\end{array}$ \\
\hline D & $\begin{array}{l}\text { augsburger- } \\
\text { allgemeine.de }\end{array}$ & $\begin{array}{l}\text { murhardter- } \\
\text { zeitung.de }\end{array}$ & $A$ & $\begin{array}{l}\text { computer- } \\
\text { welt.at }\end{array}$ & $\mathrm{CH}$ & agrihebdo.ch & $\begin{array}{l}\text { oberseezeitung. } \\
\text { ch }\end{array}$ \\
\hline D & az-online.de & mv-online.de & $A$ & dasbiber.at & $\mathrm{CH}$ & aktuell.com & $\begin{array}{l}\text { oberwiggertaler. } \\
\text { ch }\end{array}$ \\
\hline$D$ & azonline.de & mz-web.de & A & datum.at & $\mathrm{CH}$ & andelfinger.ch & obna.ch \\
\hline D & baden-online.de & n-land.de & $A$ & $\begin{array}{l}\text { derennsta- } \\
\text { ler.at }\end{array}$ & $\mathrm{CH}$ & $\begin{array}{l}\text { anzeiger-luzern. } \\
\text { ch }\end{array}$ & $\begin{array}{l}\text { obwaldner } \\
\text { zeitung.ch }\end{array}$ \\
\hline D & $\begin{array}{l}\text { badische- } \\
\text { zeitung.de }\end{array}$ & n-tv.de & $A$ & $\begin{array}{l}\text { derrostent- } \\
\text { aler.at }\end{array}$ & $\mathrm{CH}$ & anzeigerbern.ch & $\begin{array}{l}\text { oltnertagblatt. } \\
\text { ch }\end{array}$ \\
\hline D & $\begin{array}{l}\text { badisches- } \\
\text { tagblatt.de }\end{array}$ & ndaktuell.de & $A$ & $\begin{array}{l}\text { dersonntag. } \\
\text { erzdioezese- } \\
\text { wien.at }\end{array}$ & $\mathrm{CH}$ & $\begin{array}{l}\text { anzeigerkerzers. } \\
\text { ch }\end{array}$ & $\begin{array}{l}\text { ostschweiz- } \\
\text { am-sonntag.ch }\end{array}$ \\
\hline
\end{tabular}




\begin{tabular}{|c|c|c|c|c|c|c|c|}
\hline Land & URL & URL & Land & URL & Land & URL & URL \\
\hline D & $\begin{array}{l}\text { bayerische- } \\
\text { staatszeitung.de }\end{array}$ & ndr.de & A & $\begin{array}{l}\text { derstandard. } \\
\text { at }\end{array}$ & $\mathrm{CH}$ & $\begin{array}{l}\text { anzeigerkonol } \\
\text { fingen.ch }\end{array}$ & persoenlich.com \\
\hline D & bbv-net.de & ndz.de & A & diekrone.at & $\mathrm{CH}$ & $\begin{array}{l}\text { anzeigermichel } \\
\text { samt.ch }\end{array}$ & $\begin{array}{l}\text { phone.zofinger } \\
\text { tagblatt.ch }\end{array}$ \\
\hline D & bento.de & $\begin{array}{l}\text { neckar-chro- } \\
\text { nik.de }\end{array}$ & A & $\begin{array}{l}\text { diepresse. } \\
\text { com }\end{array}$ & $\mathrm{CH}$ & anzeigertgo.ch & reussbote.ch \\
\hline D & $\begin{array}{l}\text { beobachter- } \\
\text { online.de }\end{array}$ & neuepresse.de & A & e-media.at & $\mathrm{CH}$ & $\begin{array}{l}\text { anzeigervomrot- } \\
\text { tal.ch }\end{array}$ & rheintaler.ch \\
\hline D & $\begin{array}{l}\text { berchtesgade } \\
\text { ner-anzeiger.de }\end{array}$ & $\begin{array}{l}\text { neu- } \\
\text { es-deutsch- } \\
\text { land.de }\end{array}$ & A & echoonline.at & $\mathrm{CH}$ & $\begin{array}{l}\text { anzeigervon } \\
\text { saanen.ch }\end{array}$ & rontaler.ch \\
\hline D & $\begin{array}{l}\text { bergedorfer-zei- } \\
\text { tung.de }\end{array}$ & nez.de & A & $\begin{array}{l}\text { echosalz- } \\
\text { burg.at }\end{array}$ & $\mathrm{CH}$ & $\begin{array}{l}\text { appenzeller } \\
\text { zeitung.ch }\end{array}$ & rsi.ch/news \\
\hline D & $\begin{array}{l}\text { berliner-kurier. } \\
\text { de }\end{array}$ & nnn.de & A & $\begin{array}{l}\text { eibischzu- } \\
\text { ckerl.at }\end{array}$ & $\mathrm{CH}$ & arcinfo.ch & rtr.ch \\
\hline D & $\begin{array}{l}\text { berliner-zeitung. } \\
\text { de }\end{array}$ & nnp.de & A & ennsseiten.at & $\mathrm{CH}$ & avwa.ch & rts.ch \\
\hline D & $\begin{array}{l}\text { bietigheimerzei- } \\
\text { tung.de }\end{array}$ & $\begin{array}{l}\text { nordbayeri- } \\
\text { scher- } \\
\text { kurier.de }\end{array}$ & A & falter.at & $\mathrm{CH}$ & awp.ch & rzag.ch \\
\hline D & bild.de & nordbayern.de & A & $\begin{array}{l}\text { forbesdach. } \\
\text { com }\end{array}$ & $\mathrm{CH}$ & $\begin{array}{l}\text { badenertag } \\
\text { blatt.ch }\end{array}$ & saez.ch \\
\hline D & bildderfrau.de & $\begin{array}{l}\text { norder- } \\
\text { neyer-bade- } \\
\text { zeitung.de }\end{array}$ & A & furche.at & $\mathrm{CH}$ & baernerbaer.ch & saldo.ch \\
\hline D & bkz.de & nordkurier.de & A & futurezone.at & $\mathrm{CH}$ & bantigerpost.ch & $\begin{array}{l}\text { sarganserlaen } \\
\text { der.ch }\end{array}$ \\
\hline D & $\begin{array}{l}\text { bleckeder- } \\
\text { zeitung.de }\end{array}$ & $\begin{array}{l}\text { nordsee-zei- } \\
\text { tung.de }\end{array}$ & A & $\begin{array}{l}\text { ganzewoche. } \\
\text { at }\end{array}$ & $\mathrm{CH}$ & barni-post.ch & $\begin{array}{l}\text { schaffhauser- } \\
\text { nachrichten.ch }\end{array}$ \\
\hline D & bnn.de & noz.de & A & gewinn.com & $\mathrm{CH}$ & $\begin{array}{l}\text { basellandschaft- } \\
\text { lichezeitung.ch }\end{array}$ & $\begin{array}{l}\text { schweizamwo- } \\
\text { chenende.ch }\end{array}$ \\
\hline D & $\begin{array}{l}\text { boehme-zeitung. } \\
\text { de }\end{array}$ & np-coburg.de & A & gmx.at & $\mathrm{CH}$ & $\begin{array}{l}\text { bauernzeitung. } \\
\text { ch }\end{array}$ & $\begin{array}{l}\text { schweizerbauer. } \\
\text { ch }\end{array}$ \\
\hline D & $\begin{array}{l}\text { boersen-zeitung. } \\
\text { de }\end{array}$ & nq-online.de & A & grazer.at & $\mathrm{CH}$ & bazonline.ch & seetalerbote.ch \\
\hline D & $\begin{array}{l}\text { borkenerzeitung. } \\
\text { de }\end{array}$ & nrz.de & A & $\begin{array}{l}\text { handelszei- } \\
\text { tung.at }\end{array}$ & $\mathrm{CH}$ & beobachter.ch & $\begin{array}{l}\text { sempacherwo- } \\
\text { che.ch }\end{array}$ \\
\hline D & $\begin{array}{l}\text { borkumer- } \\
\text { zeitung.de }\end{array}$ & ntz.de & A & heute.at & $\mathrm{CH}$ & $\begin{array}{l}\text { bernerland } \\
\text { bote.ch }\end{array}$ & shaz.ch \\
\hline D & $\begin{array}{l}\text { boyens-medien. } \\
\text { de }\end{array}$ & nw.de & A & horizont.at & $\mathrm{CH}$ & $\begin{array}{l}\text { berneroberlaen- } \\
\text { der.ch }\end{array}$ & shn.ch \\
\hline D & br.de & nwzonline.de & A & $\begin{array}{l}\text { industrie } \\
\text { magazin.at }\end{array}$ & $\mathrm{CH}$ & bernerzeitung.ch & $\begin{array}{l}\text { simmental } \\
\text { zeitung.ch }\end{array}$ \\
\hline D & $\begin{array}{l}\text { braunschweiger- } \\
\text { zeitung.de }\end{array}$ & $\begin{array}{l}\text { oberhessische- } \\
\text { zeitung.de }\end{array}$ & A & kathpress.at & $\mathrm{CH}$ & $\begin{array}{l}\text { beundnertag- } \\
\text { blatt.ch }\end{array}$ & $\begin{array}{l}\text { solothurner } \\
\text { woche.ch }\end{array}$ \\
\hline
\end{tabular}




\begin{tabular}{|c|c|c|c|c|c|c|c|}
\hline Land & URL & URL & Land & URL & Land & URL & URL \\
\hline D & brv-zeitung.de & obermain.de & $A$ & kirchebunt.at & $\mathrm{CH}$ & bielbienne.com & $\begin{array}{l}\text { solothurner } \\
\text { zeitung.ch }\end{array}$ \\
\hline$D$ & bunte.de & on-online.de & $A$ & $\begin{array}{l}\text { kirchen.net/ } \\
\text { rupertusblatt }\end{array}$ & $\mathrm{CH}$ & bielertagblatt.ch & $\begin{array}{l}\text { sonntags } \\
\text { zeitung.ch }\end{array}$ \\
\hline$D$ & $\begin{array}{l}\text { butzbacher- } \\
\text { zeitung.de }\end{array}$ & onetz.de & $A$ & $\begin{array}{l}\text { kirchenzei } \\
\text { tung.at }\end{array}$ & $\mathrm{CH}$ & bilan.ch & sport.ch \\
\hline D & $\begin{array}{l}\text { buzzfeed.com/ } \\
\text { de/news }\end{array}$ & op-marburg.de & $A$ & $\begin{array}{l}\text { kleinezeitung. } \\
\text { at }\end{array}$ & $\mathrm{CH}$ & bilanz.ch & srf.ch/news \\
\hline$D$ & bz-berlin.de & op-online.de & $A$ & konsument.at & $\mathrm{CH}$ & blick.ch & $\begin{array}{l}\text { st-galler-nach- } \\
\text { richten.ch }\end{array}$ \\
\hline D & $\begin{array}{l}\text { cannstatter- } \\
\text { zeitung.de }\end{array}$ & $\begin{array}{l}\text { ostfriesischer- } \\
\text { kurier.de }\end{array}$ & $A$ & krone.at & $\mathrm{CH}$ & blickamabend.ch & $\begin{array}{l}\text { stadt-anzeiger. } \\
\text { ch }\end{array}$ \\
\hline D & $\begin{array}{l}\text { cellesche- } \\
\text { zeitung.de }\end{array}$ & $\begin{array}{l}\text { ostheimer } \\
\text { zeitung.de }\end{array}$ & $A$ & kurier.at & $\mathrm{CH}$ & bluewin.ch & $\begin{array}{l}\text { stadtanzei- } \\
\text { ger-olten.ch }\end{array}$ \\
\hline$D$ & chip.de & $\begin{array}{l}\text { ostsee-zeitung. } \\
\text { de }\end{array}$ & $A$ & laolal.at & $\mathrm{CH}$ & bockonline.ch & $\begin{array}{l}\text { suedostschweiz. } \\
\text { ch }\end{array}$ \\
\hline D & cn-online.de & otz.de & $A$ & $\begin{array}{l}\text { leobennews. } \\
\text { at }\end{array}$ & $\mathrm{CH}$ & $\begin{array}{l}\text { bodensee-nach- } \\
\text { richten.ch }\end{array}$ & surentaler.ch \\
\hline$D$ & come-on.de & ov-online.de & $A$ & $\begin{array}{l}\text { lustaufsle- } \\
\text { ben.at }\end{array}$ & $\mathrm{CH}$ & bote-online.ch & $\begin{array}{l}\text { surseerwoche. } \\
\text { ch }\end{array}$ \\
\hline$D$ & computerbild.de & ovb-online.de & A & martinus.at & $\mathrm{CH}$ & bote.ch & swissinfo.ch \\
\hline D & de.yahoo.com & oz-online.de & $A$ & medianet.at & $\mathrm{CH}$ & $\begin{array}{l}\text { bremgarterbe- } \\
\text { zirksanzeiger.ch }\end{array}$ & tagblatt.ch \\
\hline D & derpatriot.de & paz-online.de & $A$ & $\begin{array}{l}\text { medienmana- } \\
\text { ger.at }\end{array}$ & $\mathrm{CH}$ & $\begin{array}{l}\text { buemplizwoche. } \\
\text { ch }\end{array}$ & $\begin{array}{l}\text { tagblattzuerich. } \\
\text { ch }\end{array}$ \\
\hline D & derwesten.de & $\begin{array}{l}\text { pfaelzischer- } \\
\text { merkur.de }\end{array}$ & $A$ & megaphon.at & $\mathrm{CH}$ & bzbasel.ch & $\begin{array}{l}\text { tagesanzeiger. } \\
\text { ch }\end{array}$ \\
\hline D & dewezet.de & $\begin{array}{l}\text { pirmasener- } \\
\text { zeitung.de }\end{array}$ & $A$ & meinbezirk.at & $\mathrm{CH}$ & caffe.ch & tdg.ch \\
\hline D & die-glocke.de & pnn.de & $A$ & monat.at & $\mathrm{CH}$ & canalalpha.ch & tdg.ch \\
\hline$D$ & die-tagespost.de & pnp.de & A & monatliche.at & $\mathrm{CH}$ & cash.ch & tele1.ch/news \\
\hline D & dieharke.de & pz-news.de & $A$ & $\begin{array}{l}\text { msn.com/ } \\
\text { de-at }\end{array}$ & $\mathrm{CH}$ & cash.ch & $\begin{array}{l}\text { telebaern.tv/ } \\
\text { news }\end{array}$ \\
\hline D & diewelt.de & $\begin{array}{l}\text { remszeitung. } \\
\text { de }\end{array}$ & $A$ & $\begin{array}{l}\text { nachrichten. } \\
\text { at }\end{array}$ & $\mathrm{CH}$ & cdt.ch & telebasel.ch \\
\hline$D$ & dk-online.de & rga-online.de & $A$ & neue.at & $\mathrm{CH}$ & chomerbŠr.ch & telebielingue.ch \\
\hline D & dnn.de & rheiderland.de & $A$ & news.orf.at & $\mathrm{CH}$ & $\begin{array}{l}\text { computerworld. } \\
\text { ch }\end{array}$ & teleml.ch/news \\
\hline D & $\begin{array}{l}\text { donau-zeitung. } \\
\text { de }\end{array}$ & $\begin{array}{l}\text { rhein-zeitung. } \\
\text { de }\end{array}$ & $A$ & $\begin{array}{l}\text { news.puls4. } \\
\text { com }\end{array}$ & $\mathrm{CH}$ & derbund.ch & teleticino.ch \\
\hline D & donaukurier.de & $\begin{array}{l}\text { rheingau-echo. } \\
\text { de }\end{array}$ & $A$ & noen.at & $\mathrm{CH}$ & $\begin{array}{l}\text { digitalabo.thu- } \\
\text { nertagblatt.ch }\end{array}$ & $\begin{array}{l}\text { tessinerzeitung. } \\
\text { ch }\end{array}$ \\
\hline
\end{tabular}




\begin{tabular}{|c|c|c|c|c|c|c|c|}
\hline Land & URL & URL & Land & URL & Land & URL & URL \\
\hline D & echo-online.de & $\begin{array}{l}\text { rhoenundsaale } \\
\text { post.de }\end{array}$ & $A$ & $\begin{array}{l}\text { osttiroler- } \\
\text { bote.at }\end{array}$ & $\mathrm{CH}$ & dregion.ch & $\begin{array}{l}\text { thurgauer- } \\
\text { nachrichten.ch }\end{array}$ \\
\hline D & $\begin{array}{l}\text { einbecker- } \\
\text { morgenpost.de }\end{array}$ & $\begin{array}{l}\text { rhoenund } \\
\text { streubote.de }\end{array}$ & A & $\begin{array}{l}\text { pannonische. } \\
\text { at }\end{array}$ & $\mathrm{CH}$ & e-journal.ch & $\begin{array}{l}\text { thurgauer } \\
\text { zeitung.ch }\end{array}$ \\
\hline D & ejz.de & rnz.de & A & profil.at & $\mathrm{CH}$ & $\begin{array}{l}\text { echovongrindel- } \\
\text { wald.ch }\end{array}$ & ticinonews.ch \\
\hline D & emderzeitung.de & rp-online.de & A & regionews.at & $\mathrm{CH}$ & $\begin{array}{l}\text { einsiedler } \\
\text { anzeiger.ch }\end{array}$ & tio.ch \\
\hline D & $\begin{array}{l}\text { esslinger- } \\
\text { zeitung.de }\end{array}$ & rtl.de/news & A & $\begin{array}{l}\text { rund- } \\
\text { schau-medien. } \\
\text { at }\end{array}$ & $\mathrm{CH}$ & $\begin{array}{l}\text { engadinerpost. } \\
\text { ch }\end{array}$ & $\begin{array}{l}\text { toggenburger- } \\
\text { zeitung.ch }\end{array}$ \\
\hline D & eurosport.de & $\begin{array}{l}\text { ruhrnachrich } \\
\text { ten.de }\end{array}$ & A & rzg.at & $\mathrm{CH}$ & $\begin{array}{l}\text { entlebucher- } \\
\text { anzeiger.ch }\end{array}$ & $\begin{array}{l}\text { toggenburger } \\
\text { tagblatt.ch }\end{array}$ \\
\hline D & express.de & $\begin{array}{l}\text { rundschau- } \\
\text { online.de }\end{array}$ & A & salzburg.com & $\mathrm{CH}$ & finanzen.ch & $\begin{array}{l}\text { toponline.ch/ } \\
\text { news }\end{array}$ \\
\hline D & express.de & $\begin{array}{l}\text { saarbrue- } \\
\text { cker-zeitung. } \\
\text { de }\end{array}$ & A & salzburg24.at & $\mathrm{CH}$ & finews.ch & $\begin{array}{l}\text { triengeran } \\
\text { zeiger.ch }\end{array}$ \\
\hline D & faz.net & $\begin{array}{l}\text { saechsische. } \\
\text { de }\end{array}$ & A & $\begin{array}{l}\text { salzburger- } \\
\text { fenster.at }\end{array}$ & $\mathrm{CH}$ & franc-mont.ch & tvo-online.ch \\
\hline D & fehmarn24.de & sat1.de/news & A & salzi.at & $\mathrm{CH}$ & $\begin{array}{l}\text { frauenfelder- } \\
\text { woche.ch }\end{array}$ & $\begin{array}{l}\text { unter-emmen } \\
\text { taler.ch }\end{array}$ \\
\hline D & finanzen.net & $\begin{array}{l}\text { schiffer- } \\
\text { stadter-tag- } \\
\text { blatt.de }\end{array}$ & A & $\begin{array}{l}\text { salzkammer- } \\
\text { gut-rundblick. } \\
\text { at }\end{array}$ & $\mathrm{CH}$ & $\begin{array}{l}\text { freiburger-nach- } \\
\text { richten.ch }\end{array}$ & unterwaldner.ch \\
\hline D & $\begin{array}{l}\text { finanznachrich } \\
\text { ten.de }\end{array}$ & $\begin{array}{l}\text { schwaebi- } \\
\text { sche-post.de }\end{array}$ & A & servus.com & $\mathrm{CH}$ & $\begin{array}{l}\text { freierschweizer. } \\
\text { ch }\end{array}$ & $\begin{array}{l}\text { urnerwochen } \\
\text { blatt.ch }\end{array}$ \\
\hline D & fla.de & $\begin{array}{l}\text { schwaebische. } \\
\text { de }\end{array}$ & $A$ & servustv.com & $\mathrm{CH}$ & fricktal.info & urnerzeitung.ch \\
\hline D & flz.de & $\begin{array}{l}\text { schwarzwael- } \\
\text { der-bote.de }\end{array}$ & A & $\begin{array}{l}\text { sonntags } \\
\text { blatt.at }\end{array}$ & $\mathrm{CH}$ & fridolin.ch & vice.com/de_ch \\
\hline D & fnp.de & $\begin{array}{l}\text { schwarzwael- } \\
\text { der-post.de }\end{array}$ & $A$ & $\begin{array}{l}\text { sportzeitung. } \\
\text { at }\end{array}$ & $\mathrm{CH}$ & frutiglaender.ch & vogelgryff.ch \\
\hline D & fnweb.de & shz.de & $A$ & tagesbote.at & $\mathrm{CH}$ & fuw.ch & $\begin{array}{l}\text { volketswiler } \\
\text { nachrichten.ch }\end{array}$ \\
\hline D & focus.de & $\begin{array}{l}\text { siegener- } \\
\text { zeitung.de }\end{array}$ & $A$ & thegap.at & $\mathrm{CH}$ & gantrischpost.ch & volksstimme.ch \\
\hline D & frankenpost.de & sn-online.de & A & tips.at & $\mathrm{CH}$ & gauchebdo.ch & $\begin{array}{l}\text { wallisauerbote. } \\
\text { ch }\end{array}$ \\
\hline D & $\begin{array}{l}\text { frankfurterrund } \\
\text { schau.de }\end{array}$ & $\begin{array}{l}\text { soester- } \\
\text { anzeiger.de }\end{array}$ & A & $\begin{array}{l}\text { tirolersonn- } \\
\text { tag.at }\end{array}$ & $\mathrm{CH}$ & gdp.ch & watson.ch \\
\hline D & freiepresse.de & $\begin{array}{l}\text { solinger- } \\
\text { tageblatt.de }\end{array}$ & A & $\begin{array}{l}\text { unterkaernt } \\
\text { ner.at }\end{array}$ & $\mathrm{CH}$ & gmx.ch & $\begin{array}{l}\text { webapp-phone. } \\
\text { tagblatt.ch }\end{array}$ \\
\hline D & freitag.de & spektrum.de & $A$ & $\begin{array}{l}\text { vice.com/ } \\
\text { de_at }\end{array}$ & $\mathrm{CH}$ & $\begin{array}{l}\text { grenchnerstad- } \\
\text { tanzeiger.ch }\end{array}$ & weltwoche.ch \\
\hline
\end{tabular}




\begin{tabular}{|c|c|c|c|c|c|c|c|}
\hline Land & URL & URL & Land & URL & Land & URL & URL \\
\hline D & $\begin{array}{l}\text { freitags- } \\
\text { anzeiger.de }\end{array}$ & spiegel.de & $A$ & volksblatt.at & $\mathrm{CH}$ & $\begin{array}{l}\text { grenchnertag- } \\
\text { blatt.ch }\end{array}$ & wiggertaler.ch \\
\hline D & $\begin{array}{l}\text { fuldaerzeitung. } \\
\text { de }\end{array}$ & sport.de & $A$ & $\begin{array}{l}\text { wannundwo. } \\
\text { at }\end{array}$ & $\mathrm{CH}$ & $\begin{array}{l}\text { handelszeitung. } \\
\text { ch }\end{array}$ & $\begin{array}{l}\text { wiler-nach- } \\
\text { richten.ch }\end{array}$ \\
\hline D & ga-online.de & sportl.de & $A$ & weekend.at & $\mathrm{CH}$ & hoefner.ch & wilerzeitung.ch \\
\hline D & gaeubote.de & sportbild.de & $A$ & $\begin{array}{l}\text { welt-der- } \\
\text { frauen.at }\end{array}$ & $\mathrm{CH}$ & hoengger.ch & $\begin{array}{l}\text { willisauerbote. } \\
\text { ch }\end{array}$ \\
\hline$D$ & gala.de & sportschau.de & $A$ & $\begin{array}{l}\text { wiener- } \\
\text { online.at }\end{array}$ & $\mathrm{CH}$ & $\begin{array}{l}\text { ilgrigioneitalia- } \\
\text { no.ch }\end{array}$ & $\begin{array}{l}\text { winterthurer- } \\
\text { zeitung.ch }\end{array}$ \\
\hline D & $\begin{array}{l}\text { gandersheimer- } \\
\text { kreisblatt.de }\end{array}$ & stern.de & $A$ & wienerin.at & $\mathrm{CH}$ & illustre.ch & $\begin{array}{l}\text { wirtschafts- } \\
\text { zeit.ch }\end{array}$ \\
\hline D & gea.de & stimme.de & $A$ & $\begin{array}{l}\text { wienerzeitung. } \\
\text { at }\end{array}$ & $\mathrm{CH}$ & informatore.net & wochen-post.ch \\
\hline D & $\begin{array}{l}\text { gelnhaeuser- } \\
\text { tageblatt.de }\end{array}$ & $\begin{array}{l}\text { stuttgarter- } \\
\text { nachrichten.de }\end{array}$ & $A$ & $\begin{array}{l}\text { wirtschafts- } \\
\text { zeit.at }\end{array}$ & $\mathrm{CH}$ & inside-it.ch & $\begin{array}{l}\text { wochen-zeitung. } \\
\text { ch }\end{array}$ \\
\hline D & $\begin{array}{l}\text { general-anzeiger- } \\
\text { bonn.de }\end{array}$ & $\begin{array}{l}\text { stuttgar- } \\
\text { ter-zeitung.de }\end{array}$ & $A$ & wn24.at & $\mathrm{CH}$ & $\begin{array}{l}\text { insideparade- } \\
\text { platz.ch }\end{array}$ & wochenblatt.ch \\
\hline D & $\begin{array}{l}\text { giessener- } \\
\text { allgemeine.de }\end{array}$ & $\begin{array}{l}\text { sueddeutsche. } \\
\text { de }\end{array}$ & $A$ & $\begin{array}{l}\text { zentrum- } \\
\text { online.at }\end{array}$ & $\mathrm{CH}$ & $\begin{array}{l}\text { journalcossonay. } \\
\text { ch }\end{array}$ & $\begin{array}{l}\text { wohleranzeiger. } \\
\text { ch }\end{array}$ \\
\hline D & $\begin{array}{l}\text { giessener- } \\
\text { anzeiger.de }\end{array}$ & suedkurier.de & $A$ & $\begin{array}{l}\text { zillertaler } \\
\text { zeitung.at }\end{array}$ & $\mathrm{CH}$ & $\begin{array}{l}\text { journalde } \\
\text { morges.ch }\end{array}$ & woz.ch \\
\hline D & $\begin{array}{l}\text { gmuender- } \\
\text { tagespost.de }\end{array}$ & svz.de & $A$ & zurzeit.at & $\mathrm{CH}$ & journaldujura.ch & wundo.ch \\
\hline D & gmx.de & swp.de & & & $\mathrm{CH}$ & $\begin{array}{l}\text { jungfrauzeitung. } \\
\text { ch }\end{array}$ & $\begin{array}{l}\text { zofinger-nach- } \\
\text { richten.ch }\end{array}$ \\
\hline D & gn-online.de & $\begin{array}{l}\text { swr.de/swr } \\
\text { aktuell }\end{array}$ & & & $\mathrm{CH}$ & kanal9.ch & $\begin{array}{l}\text { zofingertagblatt. } \\
\text { ch }\end{array}$ \\
\hline D & gnz.de & sz.de & & & $\mathrm{CH}$ & kath.ch & zsz.ch \\
\hline D & $\begin{array}{l}\text { goettinger- } \\
\text { tageblatt.de }\end{array}$ & szbz.de & & & $\mathrm{CH}$ & $\begin{array}{l}\text { klettgauerbote. } \\
\text { ch }\end{array}$ & zueriost.ch \\
\hline D & golem.de & szlz.de & & & $\mathrm{CH}$ & $\begin{array}{l}\text { kloteneranzei- } \\
\text { ger.ch }\end{array}$ & zugerpresse.ch \\
\hline D & goslarsche.de & t-online.de & & & $\mathrm{CH}$ & $\begin{array}{l}\text { konsumenten- } \\
\text { schutz.ch }\end{array}$ & zugerwoche.ch \\
\hline D & $\begin{array}{l}\text { haller-kreis } \\
\text { blatt.de }\end{array}$ & t3n.de & & & $\mathrm{CH}$ & $\begin{array}{l}\text { kreuzlinger- } \\
\text { zeitung.ch }\end{array}$ & zugerzeitung.ch \\
\hline D & hallertagblatt.de & tag24.de & & & $\mathrm{CH}$ & ktipp.ch & zuonline.ch \\
\hline D & hanauer.de & tageblatt.de & & & $\mathrm{CH}$ & labroye.ch & \\
\hline D & $\begin{array}{l}\text { handelsblatt. } \\
\text { com }\end{array}$ & tagesschau.de & & & $\mathrm{CH}$ & lacote.ch & \\
\hline D & harlinger.de & $\begin{array}{l}\text { tagesspiegel. } \\
\text { de }\end{array}$ & & & $\mathrm{CH}$ & lagruyere.ch & \\
\hline
\end{tabular}




\begin{tabular}{|c|c|c|c|c|c|c|}
\hline Land & URL & URL & Land URL & Land & URL & URL \\
\hline D & harzkurier.de & tah.de & & $\mathrm{CH}$ & laliberte.ch & \\
\hline$D$ & haz.de & taz.de & & $\mathrm{CH}$ & landanzeiger.ch & \\
\hline$D$ & $\begin{array}{l}\text { heimatzeitung. } \\
\text { de }\end{array}$ & teckbote.de & & $\mathrm{CH}$ & landbote.ch & \\
\hline$D$ & heise.de & $\begin{array}{l}\text { theeuropean. } \\
\text { de }\end{array}$ & & $\mathrm{CH}$ & $\begin{array}{l}\text { langenthaler } \\
\text { tagblatt.ch }\end{array}$ & \\
\hline$D$ & $\begin{array}{l}\text { hellweger } \\
\text { anzeiger.de }\end{array}$ & $\begin{array}{l}\text { thueringer- } \\
\text { allgemeine.de }\end{array}$ & & $\mathrm{CH}$ & laregione.ch & \\
\hline$D$ & $\begin{array}{l}\text { hersbrucker- } \\
\text { zeitung.de }\end{array}$ & tlz.de & & $\mathrm{CH}$ & latele.ch & \\
\hline$D$ & $\begin{array}{l}\text { hersfelder- } \\
\text { zeitung.de }\end{array}$ & $\begin{array}{l}\text { traunsteiner- } \\
\text { tagblatt.de }\end{array}$ & & $\mathrm{CH}$ & lecourrier.ch & \\
\hline D & heute.de & tz.de & & $\mathrm{CH}$ & lejds.ch & \\
\hline$D$ & $\begin{array}{l}\text { hildesheimer- } \\
\text { allgemeine.de }\end{array}$ & uena.de & & $\mathrm{CH}$ & lemanbleu.ch & \\
\hline$D$ & hna.de & $\begin{array}{l}\text { usinger- } \\
\text { anzeiger.de }\end{array}$ & & $\mathrm{CH}$ & lematin.ch & \\
\hline$D$ & hz.de & $\begin{array}{l}\text { verlagshaus- } \\
\text { jaumann.de }\end{array}$ & & $\mathrm{CH}$ & $\begin{array}{l}\text { lematin } \\
\text { dimanche.ch }\end{array}$ & \\
\hline$D$ & idowa.de & vice.com & & $\mathrm{CH}$ & lenouvelliste.ch & \\
\hline$D$ & ihre-az.de & vkz.de & & $\mathrm{CH}$ & $\begin{array}{l}\text { lenzburger- } \\
\text { nachrichten.ch }\end{array}$ & \\
\hline$D$ & infranken.de & $\begin{array}{l}\text { vogtland- } \\
\text { anzeiger.de }\end{array}$ & & $\mathrm{CH}$ & leregional.ch & \\
\hline$D$ & $\begin{array}{l}\text { insuedthuerin- } \\
\text { gen.de }\end{array}$ & volksfreund.de & & $\mathrm{CH}$ & letemps.ch & \\
\hline$D$ & ivz-aktuell.de & $\begin{array}{l}\text { volksstimme. } \\
\text { de }\end{array}$ & & $\mathrm{CH}$ & $\begin{array}{l}\text { limmattalerzei- } \\
\text { tung.ch }\end{array}$ & \\
\hline$D$ & $\begin{array}{l}\text { jeversches-wo- } \\
\text { chenblatt.de }\end{array}$ & wa.de & & $\mathrm{CH}$ & limmatwelle.ch & \\
\hline$D$ & jungewelt.de & waddische.de & & $\mathrm{CH}$ & linthzeitung.ch & \\
\hline$D$ & $\begin{array}{l}\text { kevelaerer-blatt. } \\
\text { de }\end{array}$ & watson.de & & $\mathrm{CH}$ & lokalinfo.ch & \\
\hline D & kn-online.de & waz-online.de & & $\mathrm{CH}$ & Iqj.ch & \\
\hline$D$ & $\begin{array}{l}\text { kreis-anzeiger. } \\
\text { de }\end{array}$ & waz.de & & $\mathrm{CH}$ & $\begin{array}{l}\text { luzerner-rund- } \\
\text { schau.ch }\end{array}$ & \\
\hline D & $\begin{array}{l}\text { kreiszeitung- } \\
\text { wesermarsch.de }\end{array}$ & wdr.de & & $\mathrm{CH}$ & $\begin{array}{l}\text { luzernerzeitung. } \\
\text { ch }\end{array}$ & \\
\hline$D$ & kreiszeitung.de & welt.de & & $\mathrm{CH}$ & m.20min.ch & \\
\hline D & krzbb.de & $\begin{array}{l}\text { werra-rund- } \\
\text { schau.de }\end{array}$ & & $\mathrm{CH}$ & m.bote.ch & \\
\hline
\end{tabular}




\begin{tabular}{|c|c|c|c|c|c|c|}
\hline Land & URL & URL & Land URL & Land & URL & URL \\
\hline$D$ & ksta.de & $\begin{array}{l}\text { weser-kurier. } \\
\text { de }\end{array}$ & & $\mathrm{CH}$ & m.tio.ch & \\
\hline D & $\begin{array}{l}\text { lahrer-zeitung. } \\
\text { de }\end{array}$ & $\begin{array}{l}\text { westallgaeuer- } \\
\text { zeitung.de }\end{array}$ & & $\mathrm{CH}$ & $\begin{array}{l}\text { marchanzeiger. } \\
\text { ch }\end{array}$ & \\
\hline$D$ & landeszeitung.de & $\begin{array}{l}\text { westfalen- } \\
\text { blatt.de }\end{array}$ & & $\mathrm{CH}$ & medienwoche.ch & \\
\hline$D$ & $\begin{array}{l}\text { lauterbacher- } \\
\text { anzeiger.de }\end{array}$ & $\begin{array}{l}\text { wetterauer- } \\
\text { zeitung.de }\end{array}$ & & $\mathrm{CH}$ & $\begin{array}{l}\text { migrosmagazin. } \\
\text { ch }\end{array}$ & \\
\hline D & leinetal24.de & $\begin{array}{l}\text { wiesbadener- } \\
\text { kurier.de }\end{array}$ & & $\mathrm{CH}$ & $\begin{array}{l}\text { migrosmaga } \\
\text { zine.ch }\end{array}$ & \\
\hline$D$ & $\begin{array}{l}\text { leonberger- } \\
\text { kreiszeitung.de }\end{array}$ & $\begin{array}{l}\text { wiesbadener- } \\
\text { tagblatt.de }\end{array}$ & & $\mathrm{CH}$ & $\begin{array}{l}\text { mobile.appen } \\
\text { zellerzeitung.ch }\end{array}$ & \\
\hline$D$ & Ikz.de & $\begin{array}{l}\text { winsener- } \\
\text { anzeiger.de }\end{array}$ & & $\mathrm{CH}$ & $\begin{array}{l}\text { mobile.luzerner- } \\
\text { zeitung.ch }\end{array}$ & \\
\hline D & $\begin{array}{l}\text { In-medienhaus. } \\
\text { de }\end{array}$ & wiwo.de & & $\mathrm{CH}$ & $\begin{array}{l}\text { mobile.zuger- } \\
\text { zeitung.ch }\end{array}$ & \\
\hline$D$ & Ir-online.de & wlz-online.de & & $\mathrm{CH}$ & $\begin{array}{l}\text { mobile2.bazon- } \\
\text { line.ch }\end{array}$ & \\
\hline D & Ivz.de & wnoz.de & & $\mathrm{CH}$ & $\begin{array}{l}\text { mobile2.berner- } \\
\text { zeitung.ch }\end{array}$ & \\
\hline$D$ & Iz.de & $\begin{array}{l}\text { wolfsburger- } \\
\text { nachrichten.de }\end{array}$ & & $\mathrm{CH}$ & $\begin{array}{l}\text { mobile2.derbund. } \\
\text { ch }\end{array}$ & \\
\hline$D$ & main-echo.de & wr.de & & $\mathrm{CH}$ & $\begin{array}{l}\text { mobile2.land- } \\
\text { bote.ch }\end{array}$ & \\
\hline$D$ & mainpost.de & $\begin{array}{l}\text { wuemme- } \\
\text { zeitung.de }\end{array}$ & & $\mathrm{CH}$ & $\begin{array}{l}\text { mobile2.lematin. } \\
\text { ch }\end{array}$ & \\
\hline$D$ & $\begin{array}{l}\text { manager-maga- } \\
\text { zin.de }\end{array}$ & wz-net.de & & $\mathrm{CH}$ & $\begin{array}{l}\text { mobile2.lematin. } \\
\text { ch }\end{array}$ & \\
\hline D & maz-online.de & wz.de & & $\mathrm{CH}$ & $\begin{array}{l}\text { mobile2.tages- } \\
\text { anzeiger.ch }\end{array}$ & \\
\hline$D$ & mdr.de & wzonline.de & & $\mathrm{CH}$ & mobile2.tdg.ch & \\
\hline$D$ & merkur.de & zak.de & & $\mathrm{CH}$ & msn.com/de-ch & \\
\hline D & $\begin{array}{l}\text { mindelheimer- } \\
\text { zeitung.de }\end{array}$ & $\begin{array}{l}\text { zdf.de/ } \\
\text { nachrichten }\end{array}$ & & $\mathrm{CH}$ & murtenbieter.ch & \\
\hline$D$ & $\begin{array}{l}\text { mittelbayerische. } \\
\text { de }\end{array}$ & zeit.de & & $\mathrm{CH}$ & nau.ch & \\
\hline$D$ & mittelhessen.de & zeitonline.de & & $\mathrm{CH}$ & nau.ch & \\
\hline D & $\begin{array}{l}\text { mittelschw } \\
\text { Šbische-nach- } \\
\text { richten.de }\end{array}$ & $\begin{array}{l}\text { zevener-zei- } \\
\text { tung.de }\end{array}$ & & $\mathrm{CH}$ & news.ch & \\
\hline$D$ & mopo.de & zvw.de & & $\mathrm{CH}$ & nfz.ch & \\
\hline
\end{tabular}


